\title{
Biogeochemical roles of planktonic microbial populations in a meromictic acidic pit lake revealed by metagenomics and metatranscriptomics
}

\author{
DIANA AYALA ${ }^{1}$, JENNIFER MACALADY ${ }^{2}$, JAVIER \\ SÁNCHEZ-ESPAÑA ${ }^{3}$, CARMEN FALAGÁN $^{4}$, ESTELLE \\ COURADEAU ${ }^{1}$ AND WILLIAM BURGOS ${ }^{2}$ \\ ${ }^{1}$ The Pennsylvania State University \\ ${ }^{2}$ Pennsylvania State University \\ ${ }^{3}$ IGME \\ ${ }^{4}$ University of Exeter \\ Presenting Author: diaayala@gmail.com
}

Cueva de la Mora (CM) is a permanently stratified acidic pit lake with stable conditions to study in situ active biogeochemical processes occurring in the water column. We used metagenomics and metatranscriptomics to study the active planktonic microbial populations in the upper layer, chemocline, and deep layer of the lake. The predominant fixers of inorganic carbon in the upper layer and chemocline were Eukaryotes affiliated with the phylum Chlorophyta mainly from the genus Coccomyxa. Such primary production contributed to the activity of sulfate and iron reducers in the chemocline and deep layer. Among the bacterial sulfate reducers, members from two genera, Desulfomonile and Candidatus Desulfobacterota were highly active and abundant in the chemocline. In contrast, the deep layer possessed other uncultured taxa, mainly from the Actinobacteria, Chloroflexi, and Nitrospirae phyla, contributing to sulfur cycling. These taxa were not as abundant as taxa related to the family Thermoplasmatales, which were the most abundant populations in the deep layer. Evidence of active contributions of uncultured Thermoplasmatales to the sulfur cycling was also found. Compared to the upper layer, the chemocline possessed higher activity for iron(II) oxidation with taxa related to $\mathrm{Ca}$. Desulfobacterota, Ferrovum, Leptospirillium, and Armatimonadetes as the main iron(II) oxidizers. Activity for iron(III) reduction was only found in the deep layer affiliated with the phyla Proteobacteria. Activity for nitrogen cycling was mainly related to assimilation, although evidence for nitrogen fixation and nitrate reduction was found in the chemocline and deep layer. Overall, the oxic upper layer and chemocline have relatively high nutrients leading to the abundance of green algae. Settling algal biomass from these layers supports the microbial community in the anoxic deep layer. 